\title{
BMJ Open Efficacy of a glucagon-like peptide-1 agonist and restrictive versus liberal oxygen supply in patients undergoing coronary artery bypass grafting or aortic valve replacement: study protocol for a 2-by-2 factorial designed, randomised clinical trial
}

Sebastian Wiberg (D) , ${ }^{1}$ Jesper Kjaergaard, ${ }^{1}$ Rasmus Møgelvang, ${ }^{1}$ Christian Holdflod Møller, ${ }^{2}$ Kristian Kandler, ${ }^{2}$ Hanne Ravn (D) , ${ }^{3}$ Christian Hassager (D) , ${ }^{1}$ Lars Køber, ${ }^{1}$ Jens Christian Nilsson ${ }^{3}$

To cite: Wiberg S, Kjaergaard J, Møgelvang R, et al. Efficacy of a glucagon-like peptide- 1 agonist and restrictive versus liberal oxygen supply in patients undergoing coronary artery bypass grafting or aortic valve replacement: study protocol for a 2-by-2 factorial designed, randomised clinical trial. BMJ Open 2021;11:e052340. doi:10.1136/ bmjopen-2021-052340

- Prepublication history for this paper is available online. To view these files, please visit the journal online (http://dx.doi. org/10.1136/bmjopen-2021052340).

Received 18 April 2021 Accepted 18 October 2021

D) Check for updates

(c) Author(s) (or their employer(s)) 2021. Re-use permitted under CC BY-NC. No commercial re-use. See rights and permissions. Published by BMJ.

For numbered affiliations see end of article.

\section{Correspondence to}

Dr Sebastian Wiberg;

Sebastian.christoph.wiberg@ regionh.dk

\section{ABSTRACT}

Introduction Coronary artery bypass grafting (CABG) and/or aortic valve replacement (AVR) are associated with risk of death, as well as brain, heart and kidney injury. Glucagon-like peptide-1 (GLP-1) analogues are approved for treatment of type 2 diabetes, and GLP-1 analogues have been suggested to have potential organ-protective and anti-inflammatory effects. During cardiopulmonary bypass (CPB), consensus on the optimal fraction of oxygen is lacking. The objective of this study is to determine the efficacy of the GLP-1-analogue exenatide versus placebo and restrictive oxygenation (50\% fractional inspired oxygen, Fi02) versus liberal oxygenation (100\% Fi02) in patients undergoing open heart surgery.

Methods and analysis A randomised, placebo-controlled, double blind (for the exenatide intervention)/single blind (for the oxygenation strategy), $2 \times 2$ factorial designed single-centre trial on adult patients undergoing elective or subacute $C A B G$ and/or surgical AVR. Patients will be randomised in a 1:1 and 1:1 ratio to a 6 -hour and $15 \mathrm{~min}$ infusion of $17.4 \mu \mathrm{g}$ of exenatide or placebo during CPB and to a FiO2 of $50 \%$ or $100 \%$ during and after weaning from CPB. Patients will be followed until 12 months after inclusion of the last participant. The primary composite endpoint consists of time to first event of death, renal failure requiring renal replacement therapy, hospitalisation for stroke or heart failure. In addition, the trial will include predefined sub-studies applying more advanced measures of cardiac- and pulmonary dysfunction, renal dysfunction and cerebral dysfunction. The trial is event driven and aims at 323 primary endpoints with a projected inclusion of 1400 patients.

Ethics and dissemination Eligible patients will provide informed, written consent prior to randomisation. The trial is approved by the local ethics committee and is conducted in accordance with Danish legislation and the Declaration of Helsinki. The results will be presented in peer-reviewed journals.

\section{Strengths and limitations of this study}

- First clinical study to investigate the efficacy of a glucagon-like peptide- 1 analogue and restrictive oxygenation in patients undergoing open heart surgery.

- Randomised, placebo-controlled double/single blind 2-by-2 factorial trial design conducting analyses on the intention-to-treat population.

- Large trial expected to include 1400 patients.

- Setting is limited to a single centre.

Trial registration number NCT02673931.

\section{INTRODUCTION}

In modern management of coronary artery disease (CAD), coronary artery bypass grafting $(\mathrm{CABG})$ is often indicated, and in management of aortic stenosis, aortic valve replacement (AVR) is often applied. Both procedures require the use of cardiopulmonary bypass $(\mathrm{CPB})$. During $\mathrm{CPB}$, blood is exposed to artificial surfaces and mechanical stress potentially resulting in risk of arterial emboli and a systemic inflammatory response syndrome (SIRS) with the potential of organ damage and multiple organ failure. Patients with CAD have associated atherosclerotic disease and ischaemia-induced damage to the brain, kidneys and myocardium is frequent following open heart surgery. ${ }^{1-3}$

Following elective open heart surgery the 30-day mortality is $1 \%-2 \%$ for $\mathrm{CABG}^{45}$ and $4 \%$ for valve surgery. ${ }^{6}$ In elderly patients with 
reduced renal function and comorbidities, the 30-day mortality is as high as $20 \% .{ }^{4}$ Complications include severe heart failure and cardiogenic shock, graft occlusion or occlusion of coronary arteries, renal failure, stroke and/ or development of SIRS, which may be lethal.

The risk of acute kidney injury (AKI) requiring temporary dialysis after open heart surgery is $2 \%-3 \%$ dependent on the kidney function prior to surgery and patient age. Often, AKI is seen in conjunction with progression or development of heart failure and low cardiac output syndrome (LCOS) ${ }^{7}$ The risk of stroke during and after CABG is $1 \%-5 \% .{ }^{18}$ Factors associated with stroke in cardiac surgery are higher age, previous atheroscleroticassociated diseases and prolonged CPB-time. ${ }^{1}$ The risk of cognitive deficits after open heart surgery is above $50 \%$ decreasing to approximately $30 \%$ after 1 year, ${ }^{9}$ however, one study did not find any difference in cognitive decline in atherosclerotic patients undergoing $\mathrm{CABG}$ compared with atherosclerotic patients not undergoing CABG. ${ }^{10}$ Suggested risk factors for cognitive decline are higher age and duration of bypass, however, surgical technique (valve-insertion, CPB etc), equipment and deairing techniques may also be of importance. The physiological mechanisms suggested to cause cognitive decline include cerebral microembolism, SIRS and altered cerebral flow including LCOS. ${ }^{11}$ Several pharmacological interventions have been tested to mitigate cerebral damage during heart surgery; however, the success has been limited. ${ }^{12-16}$ Hence, there are no pharmacological interventions currently in use to hinder ischaemic damage during CPB.

\section{Glucagon-like peptide- 1 analogues}

Glucagon-like peptide-1 (GLP-1) analogues are incretin mimetics and thus increase insulin release and inhibit glucagon release. Several GLP-1 analogues, including exenatide, are approved for the treatment of type 2 diabetes.

\section{Pre-clinical data}

GLP-1 analogues have been suggested to have complex neuroprotective effects and anti-inflammatory properties. ${ }^{17}$ In rodent models, GLP-1 analogues have been shown to ameliorate neurological diseases such as Alzheimer's disease, ${ }^{18}$ Parkinson's disease ${ }^{19}$ and amyotrophic lateral sclerosis. ${ }^{20}$ In animal stroke models, GLP-1 analogues reduce the final infarct size. ${ }^{21-24}$ The mechanisms are suggested to be mediated by the intracellular AMP/PKA/CREB and the PI3K/Akt pathways, and to include reduced inflammation, oxidative stress and apoptosis that occur secondary to stroke. ${ }^{25}$ In models of acute myocardial infarction (MI) GLP-1 analogues reduce the infarct size. ${ }^{2627}$ While the understanding of the underlying mechanisms is incomplete, cardio protection induced by activation of GLP-1 receptors has been suggested to be mediated by a mechanism involving muscarinic receptors. ${ }^{28}$ Further, GLP-1 receptor activation has been demonstrated to oppose the effects of beta-adrenoceptor stimulation of cardiac ventricular excitability and to reduce ventricular arrhythmic potential. ${ }^{29}$

\section{Clinical data}

In humans, the GLP-1 analogue exenatide has been associated with increased myocardial salvage when initiated before revascularisation after $\mathrm{MI},{ }^{30}$ and in patients with a limited time of ischaemia, exenatide resulted in a smaller infarct size,${ }^{31}$ which has been confirmed by later trials. ${ }^{32} 33$ Importantly, exenatide has been administered to severely ill patients with ST-segment elevation MI (STEMI) ${ }^{31}$ and to patients resuscitated from out-of-hospital cardiac arrest $^{34}$ with no increased risk of adverse events (AEs).

\section{Liberal versus restrictive oxygen administration during weaning from $\mathrm{CPB}$}

When weaning from $\mathrm{CPB}$, there is currently no consensus on the optimal oxygen fraction, but a majority is offering a high oxygen ( $\mathrm{FiO} 2>50 \%$ ), which then gradually is reduced to maintain saturations $>95 \%$ before the patient is transferred to the intensive care unit (ICU).

\section{Preclinical data}

Several preclinical studies have suggested potential beneficial effects of hyperoxia in the preclinical setting. Conversely, it has been shown, that hyperoxia increases cerebral damage following brain ischaemia in cardiac arrest models of no-flow, ${ }^{35} 36$ as well as after deep hypothermic circulatory arrest. ${ }^{37}$

\section{Clinical data}

During and after $\mathrm{CPB}$, one of the main perioperative goals is to maintain end-organ oxygenation. Accordingly, high $\mathrm{FiO}_{2}$ levels have routinely been administered during and after CPB to protect against the risk of hypoxia and consequently organ ischaemia.

Hyperoxia has been investigated in several settings, and a final consensus on its hazards and benefits has not been reached. For simplicity, henceforth the term hyperoxia will be used in situations where the $\mathrm{FiO}_{2}$ is increased above $50 \%$. In addition to the avoidance of ischaemia, suggested beneficial effects of hyperoxia during cardiac surgery include preconditioning of the myocardium to better tolerate ischaemia, and a reduction in gaseous microemboli generated during CPB. ${ }^{38}{ }^{39}$ While hyperoxia has previously been suggested to reduce the risk of surgical site infection, ${ }^{40}$ contemporary results have been conflicting. Hyperoxia increases the risk of developing lung injury (hyperoxia-induced acute lung injury); however, it seems that several days of hyperoxia is a prerequisite for this, ${ }^{41}$ and the risk of lung injury caused by shorter periods of hyperoxia is presumably small. Ischaemic reperfusion injury of the lung in relation to $\mathrm{CPB}$ has been investigated in smaller trials; however, the impact of different ventilation strategies is questionable. ${ }^{42}$ Hyperoxia may be associated with an increase in systemic vascular resistance, and may not be associated with improved tissue oxygenation. ${ }^{43} 44$ Recently, hyperoxia has been associated with a reduced risk of surgical 
site infection after abdominal surgery in a meta-analysis, ${ }^{45}$ however, a large randomised controlled trial (RCT) found an association between hyperoxia and increased mortality in patients with malignancy undergoing abdominal surgery. ${ }^{46}$ A single-centre RCT and a meta-analysis found that a restrictive oxygenation strategy resulted in reduced mortality in critically ill patients admitted to the ICU, ${ }^{478}$ while a recent multicentre RCT in ICU patients found no difference in higher vs lower oxygen targets. ${ }^{49}$ In patients with ST-elevation acute MI, routine administration of oxygen was associated with increased myocardial injury in one study, ${ }^{50}$ however, another study found no effect of routine administration of oxygen in patients with suspected MI. ${ }^{51}$ Importantly, a recent small study has demonstrated, that a restrictive oxygenation strategy in patients undergoing CABG is safe. ${ }^{52}$ Further research is warranted, and the risks versus benefits of hyperoxia during $\mathrm{CPB}$ and immediately after weaning from $\mathrm{CPB}$ are unknown.

\section{Hypothesis}

1. Infusion with the GLP-1 analogue exenatide started preoperatively in patients undergoing elective or subacute CABG and/or AVR will reduce mortality and morbidity from heart, brain and kidney injury.

2. Restrictive oxygenation $\left(\mathrm{FIO}_{2}=50 \%\right)$ compared with liberal oxygenation $\left(\mathrm{FiO}_{2}=100 \%\right)$ during $\mathrm{CPB}$ and the first hour after weaning from $\mathrm{CPB}$ will reduce the mortality and morbidity from heart, brain and kidney injury, without increasing the risk of significant surgical site infection.

As no consensus on oxygenation targets exists, the two oxygenation strategies were based on expert opinion and endorsed by the trial steering committee (TSC). No substantial interaction between the two interventions are expected. ${ }^{53}$ In addition to the main hypothesis, three substudies will be a part of the trial. These substudies will investigate the effects of GLP-1 analogues and oxygenation strategy on cardioprotection and pulmonary protection, neuroprotection and renal protection, respectively.

\section{Endpoints}

Endpoint data will be collected and noted on specific electronic case report forms (CRFs). Supporting information will be provided to the endpoint adjudication committee for confirmation of events.

\section{Primary endpoint}

The efficacy of exenatide versus placebo and restrictive versus liberal oxygenation will be assessed by the time to the first occurring of the following coprimary endpoints within the follow-up period ending 12 months after inclusion of the last included participant:

1. Death from any cause.

2. The occurrence of any of the following AEs, adjudicated by an endpoint committee blinded for treatment allocation:
- Renal failure requiring any type of renal replacement therapy.

- Stroke, defined as any sign or symptom of neurological dysfunction persisting for more than 24 hours, determined by the treating physician based on clinical information like CT-scan, etc.

- New onset or worsening heart failure defined as need for mechanical circulatory support at the ICU, inability to close the sternum due to haemodynamic instability and/or need for inotropic haemodynamic support more than 48 hours after initiation of the first surgical procedure after randomisation. In addition, any admission for heart failure during follow-up after discharge from the index admission.

\section{Secondary endpoints}

1. Time in days to occurrence of each individual endpoint, within the follow-up period:

- Time to death from any cause.

- Time to AKI requiring renal replacement therapy.

- Time to stroke.

- Time to rehospitalisation for heart failure, or time to new onset or worsening in-hospital heart failure.

2. Incidence of any of the following safety endpoints:

- Surgical site infections with need for antibiotics for more than 48 hours (excluding routine use of antibiotics for open sternum, surgical intervention and/or endocarditis within 6 months of surgery.

- Doubling of S-creatinine or urine output below $0.5 \mathrm{~mL} / \mathrm{kg} /$ hour for 12 hours or more at any time point during index admission.

- Hypoglycaemic, defined as blood glucose $<3 \mathrm{mmol} / \mathrm{L}$, during index admission.

- Pancreatitis, defined as s-amylase $>3$ times upper normal limit, during index admission.

- A relative reduction of left ventricular ejection fraction (LVEF) of $50 \%$ compared with baseline at any time point during index admission.

- Reoperation for bleeding during index admission.

- Reoperation for any cause during index admission.

- Postsurgery MI (type $5 \mathrm{MI}^{54}$ ) during index admission.

- Readmission for cardiovascular causes within 12 months.

3. Change in cerebral performance category (CPC) from baseline to 12 months.

4. Change in modified Rankin Scale (mRS) from baseline to 12 months.

\section{METHODS AND ANALYSIS}

This is a randomised, placebo-controlled, double-blind (for the exenatide intervention)/single blind (for the oxygenation strategy), $2 \times 2$ factorial designed singlecentre trial on adult patients undergoing elective or subacute CABG and/or AVR. Patients are enrolled from a Danish tertiary university hospital with a catchment area of 2.2 million citizens older than 18 years of age. 
After completion of screening and baseline procedures, patients will be randomised to receive a GLP-1 analogue or placebo and restrictive or liberal oxygenation in a 1:1 and 1:1 ratio. By design, the factorial design of the trial can be used to test two different interventions, and as such, the trial can be regarded as two independent trials. Patients will be followed until 12 months after inclusion of the last participant.

\section{Patient and public involvement \\ No patient involved.}

\section{Inclusion}

Patients undergoing elective or subacute CABG and/or AVR will be eligible for screening, irrespective of other concomitant valve surgery. All patients will receive oral and written information and must sign the informed consent form, approved by the local ethics committee, prior to randomisation This is in accordance with Danish legislation. After provision of informed consent, patients will be registered in the trial database, and will be provided with a unique study ID. A trained study nurse will screen the patients according to the predefined inclusion and exclusion criteria (box 1) during their preoperative admission. If the criteria are met, the patients will be randomly allocated to one of the four allocation arms (GLP-1 analogue vs placebo and restrictive vs liberal oxygenation) via an internet-based randomisation algorithm using permuted blocks of 4,8 or 12 participants on the trial website. Randomisation will be stratified by planned AVR. In addition, baseline characteristics will be noted in the CRFs.

\section{Interventions}

\section{GLP-1 analogue versus placebo}

A GLP-1 analogue, exenatide (Byetta), or placebo will be administered as a 6 -hour and 15 min infusion starting at the time of anaesthesia immediately prior to surgery. The study drug (ie, either exenatide or placebo) is prepared by trained nurses with experience in preparation and administration of intravenous medications. The trial website lists enrolled patients by study ID, initials, social security number and randomisation allocation, that is, either exenatide or placebo. The study drug infusion kit consists of $1.5 \mathrm{~mL}$ of $20 \%$ Human Albumin to $248.5 \mathrm{~mL}$ of isotonic $\mathrm{NaCl}$, and then $25 \mu \mathrm{g}$ of exenatide (Byetta, Lilly) is added to patients allocated to the active study drug arm. The infusion kit is labelled with study ID, social security number as well as date and initials of the manufacturing nurse.

For each included patient, the infusion kit is brought to the coordinating anaesthetist by an investigator who is blinded for study drug allocation. The coordinating anaesthetist, who is blinded for study drug allocation, is responsible for delivering the correct investigational product to the operating theatre. The attending nurse of anaesthesia, also blinded for study drug allocation, is responsible for initiation of the study-drug infusion. The

\section{Box 1 Inclusion and exclusion criteria}

\section{Inclusion criteria}

1. Appropriately obtained written informed consent.

2. Age $\geq 18$ years.

3. Ischaemic heart disease requiring coronary artery bypass grafting and/or aortic valve disease requiring aortic valve replacement, irrespective of other concomitant valve surgery.

\section{Exclusion criteria}

1. Active treatment with glucagon-like peptide-1 analogues.

2. Obstructive hypertrophic cardiomyopathy, active myocarditis, constrictive pericarditis.

3. Hyperthyroidism or untreated hypothyroidism.

4. History of, or active pancreatitis.

5. Acute surgery; subacute surgery (ie, the following days) are eligible. 6. Known allergy towards exenatide/Byetta or albumin (vehicle).

7. On the urgent waiting list for a heart transplant (United Network of Organ Sharing category $1 \mathrm{~A}$ or $1 \mathrm{~B}$ or equivalent).

8. Recipient of any major organ transplant (eg, heart, lung, liver).

9. Receiving of has received cytotoxic or cytostatic chemotherapy and/ or radiation therapy for treatment of malignancy within 6 month month before randomisation.

10. Clinical evidence of current malignancy, with the exceptions of: basal or squamous cell carcinoma, cervical intraepithelial neoplasia, prostate cancer with a life expectancy of $>2.5$.

11. Currently enrolled in, or within 30 days from ending participation in other investigational drug trials for the treatment of diabetes or malignant obesity. Participation in other non-pharmacological trials is not an exclusion criteria.

12. Recent, within 3 months, history of alcohol or drug abuse disorder, based on self-report.

13. Pregnancy or currently breast feeding.

14. Any condition or situation that, in the investigator's opinion, could put the subject at significant risk, confound the trial's results or interfere with the subject's participation in the trial (specific reasons will be provided).

15. Previous participation in the present trial.

study drug infusion is initiated within 1 hour of scheduled start of surgery at a rate of $72 \mathrm{~mL} /$ hour $(0.12 \mu \mathrm{g} /$ $\mathrm{min}$ ) for $15 \mathrm{~min}$ (at a set volume of $18 \mathrm{~mL}$ ), followed by $26 \mathrm{~mL} /$ hour $(0.043 \mu \mathrm{g} / \mathrm{min})$ for 6 hours (at a set volume of $156 \mathrm{~mL}$ ). Thus, a total of $17.4 \mu \mathrm{g}$ of exenatide is administered. The infusion can be given in either a central or a peripheral intravenous line. The dosage and infusion rate were based on laboratory data and previously randomised trials of exenatide for cardio protection in STEMI patients, and of exenatide for neuroprotection in patients after out-of-hospital cardiac arrest. ${ }^{3055}$ Notably, in these two trials enrolling severely ill patients, the infusion rates used were not associated with an increased risk of AEs including severe hypoglycaemic or acute pancreatitis.

The time points for infusion start and end as well as rates for bolus and continuous infusion are described on a paper sheet along with measured blood glucose values from baseline to 12 hours. Any corrective glucose administered intravenously is documented. The specific care of the patients is at the discretion of the treating physician. If clinical signs of an allergic reaction or other 
life-threatening side effects are suspected the investigational product will be terminated immediately. If necessary, the treatment allocation can be unblinded and a standard operating procedures manual is available to all clinicians involved in the trial.

\section{Restrictive versus liberal oxygenation}

The patient is allocated to either restrictive or liberal oxygenation. The attending nurse preparing the study drug infusion kits registers the allocation of restrictive or liberal oxygenation on the infusion kit labels, by ticking a box labelled ' $\mathrm{FiO}_{2}=50 \%$ ' or a box labelled ' $\mathrm{FiO}_{2}=100 \%$ '.

The allocated oxygenation strategy is clearly communicated to the perfusionist and the anaesthetic nurse. The intervention period is defined as time on $\mathrm{CPB}\left(\mathrm{FiO}_{2}\right.$ on oxygenator) and for the first hour after weaning off $\mathrm{CPB}$ (FiO2 on the ventilator). Tidal volumes and inspiratory pressures are adjusted according to local guidelines. Positive end-expiratory pressure, peak pressure, respiratory rate (RR) and minute volumes are recorded at $5 \mathrm{~min}$ intervals. In addition, arterial blood gases are analysed at least once on $\mathrm{CPB}$ and once while ventilated during the intervention period.

Dosage adjustments are not intended. However, increasing $\mathrm{FiO}_{2}$ is allowed if arterial oxygen saturation $\left(\mathrm{SaO}_{2}\right)$ drops below $92 \%$ for more than $30 \mathrm{~s}$ or if deemed necessary to ensure the patients' safety. It is recommended, that $\mathrm{FiO}_{2}$ is reduced as soon as it is considered safe for the patient, preferably to $50 \%$. Any increase is at the treating physician's discretion, and any dose adjustments are documented along with the reasons for increasing $\mathrm{FiO}_{2}\left(\mathrm{SaO}_{2}<92 \%\right.$, arrhythmia, concern for cerebral oxygenation etc). The specific care of the patients is at the discretion of the treating physician, and information on protocol violations and/or concomitant therapy is collected on CRFs. Necessary interventions will not be delayed by the trial intervention.

\section{Assessments}

The investigator will be responsible for ensuring that all assessments are performed according to protocol, and that the data are recorded in the electronic CRFs. Specific plans for data entry and security have been described in the trial protocol. Missing data, as well as the reasons, must be reported in the CRFs. All routine laboratory analyses will be performed on point-of-care systems or at the hospital's local laboratory.

\section{Preoperative assessments}

Prior to surgery, the following variables will be noted in the CRFs:

- Verified informed consent.

- Medical history including previous surgery.

- New York Heart Association (NYHA) classification.

- Euro Score and Society of Thoracic Surgeons (STS) score (evaluated by the attending surgeon).

- CPC.

- mRS.
- Self-perceived function 'two simple questions'.

- LVEF.

- Physical examination including age, sex, height, body weight.

- Vital signs including blood pressure, heart rate (HR), RR and peripheral oxygenation.

- Any concomitant therapy.

\section{Follow-up assessments}

Follow-up assessments will occur during index admission, after 3 and 12 months as well as at end of follow-up. The 3 months assessment will be done during an in-hospital visit, whereas the other assessments will be telephone/ registry based.

At each follow-up the following will be recorded:

- The occurrence of any AE.

- The occurrence of serious AEs (SAE).

- NYHA classification.

- Patient-reported outcome questionnaires will be answered at 1 week and 3 months.

- CPC.

- mRS.

- Self-perceived function 'two simple questions'.

- Vital signs will be recorded throughout admission and at 3 months follow-up visit.

From the day after surgery all information on SAE will be recorded.

\section{Blood sampling}

Blood will be drawn for biochemistry at baseline, the morning after surgery, at day three and five after surgery and after 3 months. The following analyses will be conducted at each blood draw: Sodium, potassium, calcium, glucose, albumin, blood urea nitrogen, uric acid, bilirubin (total), creatinine, S-protein, red cell count, white cell count, platelet count, HbA1c (solely preoperatively), $\mathrm{N}$-terminal-pro-brain natriuretic peptide (NT-pro-BNP), C reactive protein. In addition, $3 \times 10 \mathrm{~mL}$ of blood will be drawn and stored in a biobank for up to 20 years.

\section{Substudy assessments}

Each of the three substudies will make individual assessments as described below.

\section{Cardio and pulmonary protection substudy}

The potential effect of the two interventions on the heart will be studied by transthoracic echocardiography in a substudy. The first 1080 included patients will be examined by advanced 2D and 3D echocardiography the day before surgery, 4-6 days after surgery (before discharge) and 3 months after surgery. Besides global, regional and layer-specific circumferential, radial, longitudinal and area strain measures, the echocardiographic assessment will include evaluation of systolic function (2dimensional LVEF, 3 dimensional LVEF/right ventricular ejection fraction (RVEF), dp/dt, mitral and tricuspid annular movement), diastolic function (left atrial volume, mitral inflow velocities, early and late diastolic mitral and tricuspid 
movements), cardiac time intervals, valve disease, pericardial effusion and constriction, and ventricular, vena caval and aortic dimensions. The primary echocardiographic endpoint regarding both interventions in the trial is global longitudinal strain. In a subset of patients, a $6 \mathrm{~min}$ hall walk test and serial 12-ECGs were also performed.

For the pulmonary protection substudy, an advanced lung function testing with DLCO is performed using the EasyOne Pro manufactured by ndd Medizintechnik AG, Technoparkstr. 1, 8005 Zürich, Switzerland, wwwnddch. The tests consist of spirometry and DLCO (dilution gas technique ( $10 \%$ helium, $0.3 \%$ carbon monoxide and $18 \%$ to $25 \%$ oxygen (normally $21 \%$ )). The tests are performed the day before surgery, before discharge and on the follow-up visit after 3 months by trained study personnel where possible in the first 800 patients. Participation in the substudy is voluntary.

The following hypothesis are investigated:

1. Exenatide infusion during cardiac surgery is associated with less decline DLCO and FEV1/FVC after 3 months compared with placebo.

2. Restrictive oxygenation during cardiac surgery is associated with less decline DLCO and FEV1/FVC after 3 months compared with placebo.

In addition, the association of advanced lung functions tests and prognosis, risk of $\mathrm{AE}$ and subsequent heart failure events are evaluated. Correlations to echocardiographic parameters of cardiac systolic and diastolic function are also evaluated.

\section{Neuroprotection substudy}

The neuroprotection substudy will be based on the biobank, measuring multiple markers of brain injury, cognitive outcomes at 3 months follow-up visit, and mRS and CPC scores. Biomarkers will be measured in the entire study cohort, whereas neurological assessment is performed in the first 1100 patients only due to limited resources. The primary aim will be associations between interventions and markers of cerebral injury measured the first days following surgery (primary endpoint), and secondary endpoints will include the relation to mRS, CPC and risk of death during the follow-up.

\section{Renoprotection substudy}

The primary outcome of the renoprotection substudy is acute kidney injury (AKI) defined according to the Kidney Disease Improving Global Outcomes criteria, which is based on S-creatinine increases and urine output. Secondary outcomes will include glomerular filtration rate (GFR) measured by Cr-EDTA clearance, estimated GFR and novel biomarkers of AKI. The primary endpoint will be based on the entire study cohort.

\section{Safety}

The trial population consists of patients with ischaemic heart disease and/or aortic valve disease, who undergo CABG and/or AVR. The majority of AEs are relatively common irrespective of treatment strategies. The occurrence of any $\mathrm{AE}$ will be recorded daily during index admission and at all follow-ups.

\section{Adverse events}

AEs are defined as undesirable medical occurrences or worsening of pre-existing medical conditions that occur after initiation of the investigational product, whether or not considered to be related to the investigational product.

\section{Serious AEs}

SAEs are defined as AEs resulting in significant side effects including ones that are fatal, life-threatening, require hospitalisation of prolongation of hospitalisation and/or are persistent or significant.

\section{Suspected unexpected serious adverse reactions}

Suspected unexpected serious adverse reactions (SUSARs) are defined as unexpected, serious AEs with presumed relation to the investigational drug. The term 'unexpected' is defined using the Byetta Summary of product characteristics (http://www.ema.europa.eu/ docs/en_GB/document_library/EPAR_-_Product_Information/human/000698/WC500051845.pdf, current version 16 August 2016) and the Conoxia Summary of product characteristics available at the Danish Medicines Agency (http://produktresume.dk/AppBuilder/ search?q=Conoxia $+100+\% 25 \% 2 \mathrm{C}+$ medicinsk + gas $\% 2 \mathrm{C}+$ komprimeret.doc, current version 19 October 2021).

\section{Reporting procedures}

Safety variables and SAEs will be recorded continuously in the CRF during the first 7 days after surgery. AEs occurring after 7 days will be recorded at the preplanned follow-up visits. All medically significant AEs considered by the investigator or the sponsor to be related to the investigational product will be followed until resolved or considered stable. The following attributes will be recorded by the investigator: description, dates of onset and resolution, severity, assessment of whether the AE is related to the investigational product, other suspected drugs or devices, and action taken. For each $\mathrm{AE}$ reported in the CRF, the investigator will adjudicate whether the event is an SAE. All SAEs will be recorded on a SAE Report form. The sponsor is responsible for reporting all SUSARs to the Danish Health and Medicines Authority as soon as possible and no later than 7 days after awareness.

\section{Data monitoring}

A data monitoring and safety committee (DMSC) has been assembled and consists of individuals free of any potential conflicts of interests. The DMSC is responsible for ensuring the interests of trial participants, for assessing safety and efficacy of the two trial interventions and for monitoring the conduct of the trial. The DMSC can recommend stopping of continuing the trial to the TSC. Also, the DMSC can formulate recommendations regarding all elements of the trial conduct, in order to enhance the trial integrity. Any recommendations from 
the DMSC regarding stopping, continuing or changing the trial will be communicated to the TSC without delay. The TSC is responsible for reviewing any recommendations from the DMSC and to determine, whether changes in trial conduct are required. The sponsor is responsible for reporting the number of SAEs and SUSARs to the DMSC bimonthly until 250 patients have been randomised. The need for further evaluation of SAEs and all-cause mortality will be decided by the DMSC. Interim analyses will be performed by a statistician selected by the DMSC.

An endpoint classification committee will adjudicate primary endpoints in a blinded fashion.

Independent good clinical practice (GCP) units will monitor informed written consent forms, data quality and adjudication of endpoints.

\section{Planned statistical analyses}

\section{Sample size estimation}

This parallel group trial investigates two interventions, and we plan to analyse these two interventions as two separate studies. The effect of the two interventions are not expected to interact, therefore, the design and sample size estimation did not account for such interaction. The study involving restrictive versus liberal oxygenation is subordinate to the study involving the GLP-1 analogue, and the potential interactions of the two interventions will be analysed in the GLP-1 analogue trial. Thus, the power calculations are based on the GLP-1 analogue intervention.

The trial is event driven, aiming at 323 primary endpoints to be able to show a $25 \%$ reduction in the primary endpoint with a power of $80 \%$ at an $\alpha$-level of 0.05 (two sided). Based on cumulative event rates from the surgical register at Rigshospitalet (unpublished), a total of 1400 patients are needed to reach a total of 323 events during follow-up. We will include 1400 patients in the trial. We will follow all patients until 323 events have been reached and the last patient has been followed for a minimum of 12 months. Accordingly, the follow-up period will vary from approximately 6 years from the earliest included patients to 12 months for the last included patient.

\section{General principles}

All analyses will be performed according to the intentionto-treat principle. ${ }^{56} \mathrm{~A}$ two-sided significance level of 0.05 will be applied throughout. Missing data will be reported in the publication. In case of more than $5 \%$ missing data in outcome variables, multiple imputation with creation of 50 imputed datasets will be analysed separately and aggregated into an estimate of the intervention's effect on the primary endpoint. ${ }^{57} 58$ For non-fatal events, competing risk of events will be accounted for.

\section{Inclusion profile}

In accordance with the Consolidated Standards of Reporting Trials diagram, ${ }^{59}$ a flow chart of the trial inclusion and exclusion profile will be provided.

\section{Baseline variables}

The following baseline variables will be included in box 1 .

1. Demographics

- Sex.

- Age per year.

- body mass index (BMI).

- History of smoking.

- Estimated amount of alcohol consumed per week per grams.

2. Medical history

- Comorbidity and function (including CPC-class, previous heart failure, previous MI, ischaemic heart disease, previous arrhythmia, previous cardiac arrest, arterial hypertension, transient ischaemic attack or stroke, epilepsy, diabetes, asthma or chronic obstructive pulmonary disease, chronic haemodialysis or peritoneal dialysis, hepatic cirrhosis, haematological malignancy, other malignancy, AIDS, alcoholism, intravenous drug abuse, or other immunodeficiency).

- Previous percutaneous coronary intervention

- Previous CABG.

- Previous aortic valve surgery.

- Implantable cardioverter-defibrillator and/or pacemaker.

- Current medical therapy at time of surgery.

3. Surgical procedure

- Indication for surgery.

- CABG.

- Number of grafts, stratified by arterial and venous grafts.

- AVR, stratified by type of valve

- Combined CABG and AVR

- Duration of surgery (minutes).

- Duration of CPB (minutes)

- Duration of aortic cross clamp time (minutes)

Baseline variables will be stratified according to treatment allocation. Continuous variables will be presented as mean \pm SD if normally distributed or otherwise as median (IQR). Differences between allocation groups will be tested with the independent sample t-test, potentially after logarithmic transformation for lognormally distributed variables, or the non-parametric Mann-Whitney U test as appropriate. Categorical variables will be presented as number (percentage) and differences between allocation groups will be tested with the chi-square test or the Fisher's exact test as appropriate.

\section{Endpoint analyses}

Primary endpoint

For each of the two interventions (exenatide and oxygenation), Kaplan-Meier curves will be graphically displayed, and compared using the two-sided log-rank test. In 
addition, a multivariable analysis of the time to first event will be performed using Cox proportional hazard models. The model will be adjusted for the following covariates: treatment allocation, age, sex, BMI, indication for surgery, year of inclusion, procedure (CABG vs AVR vs CABG+AVR), known alcohol or drug abuse, Charlson comorbidity index, previous percutaneous coronary intervention (PCI), previous CABG, previous AVR, length of procedure, length of CPB. The HR with $95 \%$ CIs will be reported. Since the primary intervention is exenatide versus placebo, the potential interactions between this treatment and the oxygenation-allocation on outcome and treatment will be estimated in the article on exenatide via the Cox proportional hazard models, with oxygenation group as a covariate in the model.

Censoring: Subjects withdrawing from the study early (other than for withdrawal of consent) will be followed for potential development of the primary endpoint. Subjects completing the study and not experiencing the composite event will be censored.

\section{Secondary endpoint}

Time to the individual secondary endpoints will be analysed with Kaplan-Meier estimates for all-cause mortality and cumulative incidence rates for other endpoints taking competing risk of death into account. The censoring mechanism will be similar to the one applied to the primary endpoint. The type 1 error rate associated with multiple comparisons will be controlled with the application of Benjamini-Hochberg adjustment. ${ }^{60}$ Differences in the occurrence of pre-defined AEs between allocation groups will be analysed with the $\chi^{2}$ test or the Fisher's exact test, as appropriate. Changes in continuous variables over time will be analysed using linear mixed models.

\section{Safety endpoint}

Incidence rates of AEs will be graded according to severity and relationship to the investigational product. Tables of deaths, serious and significant AEs, including ones causing early withdrawal will be provided. Differences in incidence of AEs, as well as the cumulative incidence of AEs between groups, will be analysed with the $\chi^{2}$ test or the Fisher's exact test, as appropriate.

\section{Ethics and dissemination}

Participation in the trial will not delay routine or therapeutic procedures. The mortality and morbidity after open heart surgery (including CABG and AVR) are mainly caused by organ failure and inflammation. Thus, methods of organ protection are considered essential for reducing the mortality after open heart surgery. In addition, increased knowledge of organ function following surgery will potentially be of benefit to patients undergoing open heart surgery.

Exenatide has been used for treatment of type 2 diabetes for years. In addition, it has been used in STEMI patients and out-of-hospital cardiac arrest patients without increased risk of AEs compared with placebo. As infusion of study drug will occur simultaneously with surgery, and the allocated oxygenation strategy will occur during CPB, when weaning from and the following hour after weaning from $\mathrm{CPB}$, the participants will experience no side effects. The organ-protective effects of exenatide and restrictive oxygenation during open heart surgery cannot be gained outside the clinical setting, and human experimental models are obviously unethical. All patients will provide oral and written informed consent prior to inclusion in the trial.

\section{Approvals}

The trial is conducted in accordance with Danish legislation and the Declaration of Helsinki.

In addition, the trial is conducted in accordance with international standards for GCP and is monitored by an independent GCP unit. The trial is surveyed by a DMSC with full access to the study database on request. The trial protocol, including any amendments and written information and consent forms have been approved prior to initiation of the trial by the local ethics committee (ref. no. H-15010562). In addition, The Danish Health and Medicines Authority approved the trial (protocol ref. HJE-PHARMA-001, EudraCT no. 2015-003050-41).

\section{Dissemination}

All results will be published in international, peerreviewed journals and presented at international congresses. Coauthorships will be granted in accordance with the Vancouver guidelines. In case the trial demonstrates a significant, positive effect of exenatide or restrictive oxygenation during heart surgery these treatment strategies will be easy to implement.

\section{Author affiliations}

${ }^{1}$ Department of Cardiology, Rigshospitalet, Copenhagen, Denmark

${ }^{2}$ Department of Cardiothoracic Surgery, Rigshospitalet, Copenhagen, Denmark

${ }^{3}$ Department of Cardiothoracic Anesthesiology, Rigshospitalet, Copenhagen, Denmark

Contributors Authors SW, JK, RM, CHM, KK, HBR, CH, LK and JCN all contributed equally to the conceptualisation of the trial or substudies. All authors have contributed to drafting and writing the protocol and substudy protocols. Author SW wrote the first draft of this design article based on the study protocol. All authors, SW, JK, RM, CHM, KK, HBR, CH, LK and JCN have read and approved the final manuscript.

Funding This work was supported by: Læge Sofus Carl Emil Friis og Hustru Olga Doris Friis' Legat. 2017 (DKR461 180), Aase og Ejnar Danielsens Fond 2017, grant no. 10-001 976 (DKR200 000), Danish Heart Foundation 2019, Grant number 19-R133-A9174-22144 (DKR1 000 000), The Heart Centre Research Foundation 2017, (DKR160 000), Lundbeck Foundation (R186-2015-2132).

\section{Competing interests None declared.}

Patient and public involvement Patients and/or the public were not involved in the design, or conduct, or reporting, or dissemination plans of this research.

Patient consent for publication Not applicable.

Provenance and peer review Not commissioned; externally peer reviewed.

Open access This is an open access article distributed in accordance with the Creative Commons Attribution Non Commercial (CC BY-NC 4.0) license, which permits others to distribute, remix, adapt, build upon this work non-commercially, and license their derivative works on different terms, provided the original work is 
properly cited, appropriate credit is given, any changes made indicated, and the use is non-commercial. See: http://creativecommons.org/licenses/by-nc/4.0/.

\section{ORCID iDs}

Sebastian Wiberg http://orcid.org/0000-0002-7062-7189

Hanne Ravn http://orcid.org/0000-0003-4702-5195

Christian Hassager http://orcid.org/0000-0002-1199-0981

\section{REFERENCES}

1 Mao Z, Zhong X, Yin J, et al. Predictors associated with stroke after coronary artery bypass grafting: a systematic review. J Neurol Sci 2015;357:1-7.

2 Wijeysundera DN, Karkouti K, Beattie WS, et al. Improving the identification of patients at risk of postoperative renal failure after cardiac surgery. Anesthesiology 2006;104:65-72.

3 Salamonsen RF, Schneider H-G, Bailey M, et al. Cardiac troponin I concentrations, but not electrocardiographic results, predict an extended hospital stay after coronary artery bypass graft surgery. Clin Chem 2005;51:40-6.

4 Houlind K, Kjeldsen BJ, Madsen SN, et al. On-Pump versus offpump coronary artery bypass surgery in elderly patients: results from the Danish on-pump versus off-pump randomization study. Circulation 2012;125:2431-9.

5 Nicolini F, Vezzani A, Fortuna D, et al. Gender differences in outcomes following isolated coronary artery bypass grafting: longterm results. J Cardiothorac Surg 2016;11:1-12.

6 Thyregod HGH, Steinbrüchel DA, Ihlemann N, et al. Transcatheter versus surgical aortic valve replacement in patients with severe aortic valve stenosis: 1-year results from the All-Comers notion randomized clinical trial. J Am Coll Cardiol 2015;65:2184-94.

7 Pannu N, Graham M, Klarenbach S, et al. A new model to predict acute kidney injury requiring renal replacement therapy after cardiac surgery. CMAJ 2016;188:1076-83.

8 Mérie C, Køber L, Olsen PS, et al. Risk of stroke after coronary artery bypass grafting. Stroke 2012:43:38-43.

9 Newman MF, Kirchner JL, Phillips-Bute B, et al. Longitudinal assessment of neurocognitive function after coronary-artery bypass surgery. N Engl J Med 2001;344:395-402.

10 Selnes OA, Grega MA, Bailey MM, et al. Do management strategies for coronary artery disease influence 6-year cognitive outcomes? Ann Thorac Surg 2009;88:445-54.

11 Cropsey C, Kennedy J, Han J, et al. Cognitive dysfunction, delirium, and stroke in cardiac surgery patients. Semin Cardiothorac Vasc Anesth 2015;19:309-17.

12 Nagels W, Demeyere R, Van Hemelrijck J, et al. Evaluation of the neuroprotective effects of $\mathrm{S}(+)$-ketamine during open-heart surgery. Anesth Analg 2004;98:1595-603

13 Taggart DP, Browne SM, Wade DT, et al. Neuroprotection during cardiac surgery: a randomised trial of a platelet activating factor antagonist. Heart 2003;89:897-900.

14 Wang D, Wu X, Li J, et al. The effect of lidocaine on early postoperative cognitive dysfunction after coronary artery bypass surgery. Anesth Analg 2002;95:1134-41.

15 Arrowsmith J, Harrison MJG, Newman SP. Neuroprotection of the brain during cardiopulmonary bypass: a randomized trial of remacemide during coronary artery bypass in 171 patients. Stroke 2015;29:2357-62.

16 Kong RS, Butterworth J, Aveling W, et al. Clinical trial of the neuroprotectant clomethiazole in coronary artery bypass graft surgery: a randomized controlled trial. Anesthesiology 2002;97:585-91.

17 Hölscher C. Potential role of glucagon-like peptide-1 (GLP-1) in neuroprotection. CNS Drugs 2012;26:871-82.

18 McClean PL, Parthsarathy V, Faivre E, et al. The diabetes drug liraglutide prevents degenerative processes in a mouse model of alzheimer's disease. J Neurosci 2011;31:6587-94.

19 Harkavyi A, Abuirmeileh A, Lever R, et al. Glucagon-like peptide 1 receptor stimulation reverses key deficits in distinct rodent models of Parkinson's disease. J Neuroinflammation 2008;5:19.

$20 \mathrm{Li}$ Y, Chigurupati S, Holloway HW, et al. Exendin-4 ameliorates motor neuron degeneration in cellular and animal models of amyotrophic lateral sclerosis. PLoS One 2012;7:e32008-13.

21 Teramoto S, Miyamoto N, Yatomi K, et al. Exendin-4, a glucagonlike peptide-1 receptor agonist, provides neuroprotection in mice transient focal cerebral ischemia. J Cereb Blood Flow Metab 2011;31:1696-705.

22 Briyal S, Gulati K, Gulati A. Repeated administration of exendin-4 reduces focal cerebral ischemia-induced infarction in rats. Brain Res 2012;1427:23-34.
23 Jiang D, Wang Y, Zang Y, et al. Neuroprotective effects of rhGLP-1 in diabetic rats with cerebral ischemia/reperfusion injury. Drug Dev Res 2016;77:124-33.

24 Zhang H, Meng J, Zhou S, et al. Intranasal delivery of exendin-4 confers neuroprotective effect against cerebral ischemia in mice. Aaps J 2016;18:385-94.

25 Marlet IR, Ölmestig JNE, Vilsbøll T, et al. Neuroprotective mechanisms of glucagon-like peptide-1-based therapies in ischaemic stroke: a systematic review based on pre-clinical studies. Basic Clin Pharmacol Toxicol 2018;122:559-69.

26 Timmers L, Henriques JPS, de Kleijn DPV, et al. Exenatide reduces infarct size and improves cardiac function in a porcine model of ischemia and reperfusion injury. J Am Coll Cardiol 2009;53:501-10.

27 Ravassa S, Zudaire A, Díez J. Glp-1 and cardioprotection: from bench to bedside. Cardiovasc Res 2012;94:316-23.

28 Basalay MV, Mastitskaya S, Mrochek A, et al. Glucagon-Like peptide-1 (GLP-1) mediates cardioprotection by remote ischaemic conditioning. Cardiovasc Res 2016;112:669-76.

29 Ang R, Mastitskaya S, Hosford PS, et al. Modulation of cardiac ventricular excitability by GLP-1 (glucagon-like peptide-1). Circ Arrhythm Electrophysiol 2018;11:e006740.

30 Lønborg J, Vejlstrup N, Kelbæk H, et al. Exenatide reduces reperfusion injury in patients with ST-segment elevation myocardial infarction. Eur Heart J 2012;33:1491-9.

31 Lønborg J, Kelbæk H, Vejlstrup N, et al. Exenatide reduces final infarct size in patients with ST-segment-elevation myocardial infarction and short-duration of ischemia. Circ Cardiovasc Interv 2012:5:288-95.

32 Woo JS, Kim W, Ha SJ, et al. Cardioprotective effects of exenatide in patients with ST-Segment-Elevation myocardial infarction undergoing primary percutaneous coronary intervention. Arterioscler Thromb Vasc Biol 2013;33:2252-60.

33 Bernink FJP, Timmers L, Diamant M, et al. Effect of additional treatment with exenatide in patients with an acute myocardia infarction: the exami study. Int J Cardiol 2013;167:289-90.

34 Wiberg S, Hassager $\mathrm{C}$, Schmidt $\mathrm{H}$, et al. Neuroprotective effects of the glucagon-like peptide-1 analog exenatide after out-ofhospital cardiac arrest: a randomized controlled trial. Circulation 2016;134:2115-24

35 Vereczki V, Martin E, Rosenthal RE, et al. Normoxic resuscitation after cardiac arrest protects against hippocampal oxidative stress, metabolic dysfunction, and neuronal death. J Cereb Blood Flow Metab 2006;26:821-35.

36 Liu Y, Rosenthal RE, Haywood Y, et al. Normoxic ventilation after cardiac arrest reduces oxidation of brain lipids and improves neurological outcome. Stroke 1998;29:1679-86.

37 Nollert G, Nagashima M, Bucerius J, et al. Oxygenation strategy and neurologic damage after deep hypothermic circulatory arrest. II. hypoxic versus free radical injury. J Thorac Cardiovasc Surg 1999;117:1172-9.

38 Heinrichs J, Lodewyks C, Neilson C, et al. The impact of hyperoxia on outcomes after cardiac surgery: a systematic review and narrative synthesis. Can J Anaesth 2018;65:923-35.

39 Young RW. Hyperoxia: a review of the risks and benefits in adult cardiac surgery. J Extra Corpor Technol 2012;44:241-9.

40 Greif R, Akça O, Horn EP, et al. Supplemental perioperative oxygen to reduce the incidence of surgical-wound infection. $N$ Engl J Med 2000;342:161-7.

41 Kallet RH, Matthay MA. Hyperoxic acute lung injury. Respir Care 2013:58:123-41.

42 Schreiber J-U, Lancé MD, de Korte M, et al. The effect of different lung-protective strategies in patients during cardiopulmonary bypass: a meta-analysis and semiquantitative review of randomized trials. J Cardiothorac Vasc Anesth 2012;26:448-54.

43 Waring WS, Thomson AJ, Adwani SH, et al. Cardiovascular effects of acute oxygen administration in healthy adults. $J$ Cardiovasc Pharmacol 2003;42:245-50.

44 Anderson KJ, Harten JM, Booth MG, et al. The cardiovascular effects of normobaric hyperoxia in patients with heart rate fixed by permanent pacemaker. Anaesthesia 2010;65:167-71.

45 Hovaguimian F, Lysakowski C, Elia N, et al. Effect of intraoperative high inspired oxygen fraction on surgical site infection, postoperative nausea and vomiting, and pulmonary function: systematic review and meta-analysis of randomized controlled trials. Anesthesiology 2013;119:303-16.

46 Meyhoff CS, Jorgensen LN, Wetterslev J, et al. Increased long-term mortality after a high perioperative inspiratory oxygen fraction during abdominal surgery: follow-up of a randomized clinical trial. Anesth Analg 2012;115:849-54.

47 Girardis M, Busani S, Damiani E, et al. Effect of conservative vs conventional oxygen therapy on mortality among patients in an 
intensive care unit: the oxygen-ICU randomized clinical trial. JAMA 2016;316:1583-7.

48 Chu DK, Kim LH-Y, Young PJ, et al. Mortality and morbidity in acutely ill adults treated with liberal versus conservative oxygen therapy (iota): a systematic review and meta-analysis. Lancet 2018;391:1693-705.

49 Schjørring OL, Klitgaard TL, Perner A, et al. Lower or higher oxygenation targets for acute hypoxemic respiratory failure. $N$ Engl $J$ Med 2021;384:1301-11.

50 Stub D, Smith K, Bernard S, et al. Air versus oxygen in ST-segmentelevation myocardial infarction. Circulation 2015;131:2143-50.

51 Hofmann R, James SK, Jernberg T, et al. Oxygen therapy in suspected acute myocardial infarction. $N$ Engl J Med 2017;377:1240-9.

52 Smit B, Smulders YM, de Waard MC, et al. Moderate hyperoxic versus near-physiological oxygen targets during and after coronary artery bypass surgery: a randomised controlled trial. Crit Care 2016;20:1-10.

53 Reade MC, Bass F, Howe B, et al. Considerations for co-enrolment in randomised controlled effectiveness trials in critical care: the SPICE-8 co-enrolment guidelines. Crit Care Resusc 2017;19:110114.
54 Thygesen K, Alpert JS, Jaffe AS, et al. Third universal definition of myocardial infarction. Eur Heart $J$ 2012;33:2551-67.

55 Wiberg S, Hassager C, Thomsen JH, et al. Glp-1 analogues for neuroprotection after out-of-hospital cardiac arrest: study protocol for a randomized controlled trial. Trials 2016;17:304.

56 Fergusson D, Aaron SD, Guyatt G, et al. Post-Randomisation exclusions: the intention to treat principle and excluding patients from analysis. BMJ 2002;325:652-4.

57 Donders ART, van der Heijden GJMG, Stijnen T, et al. Review: a gentle introduction to imputation of missing values. J Clin Epidemiol 2006;59:1087-91.

58 Schafer JL, Graham JW. Missing data: our view of the state of the art. Psychol Methods 2002;7:147-77.

59 Moher D, Schulz KF, Altman DG. The consort statement: revised recommendations for improving the quality of reports of parallelgroup randomised trials. Lancet 2001;357:1191-4.

60 Benjamini Y, Hochberg Y. Controlling the false discovery rate: a practical and powerful approach to multiple testing. Journal of the Royal Statistical Society: Series B 1995;57:289-300. 